\title{
Overcoming Challenges in the Changing Environment of Practice-Based Research
}

Stacia A. Finch, $M A^{1}$

Richard Wasserman, $M D^{1,2}$

Emara Nabi-Burza, MBBS, MS $S^{3,4}$

Bethany Hipple, MPH ${ }^{3,4}$

Robert Oldendick, $\mathrm{PbD}^{5}$

Jonathan P. Winickoff, MD, MPH $H^{3,4}$

'Pediatric Research in Office Settings, American Academy of Pediatrics, Elk Grove Village, Illinois

${ }^{2}$ Department of Pediatrics, University of Vermont, Burlington, Vermont

${ }^{3}$ Center for Child and Adolescent Health and Research Policy, Massachusetts General Hospital, Boston, Massachusetts

${ }^{4}$ Julius B. Richmond Center of Excellence, American Academy of Pediatrics, Elk Grove Village, Illinois

${ }^{5}$ University of South Carolina, Institute for Public Service and Policy Research, Columbia, South Carolina

\section{sim MORE ONLINE www.annfammed.org}

Conflicts of interest: authors report none.

\section{CORRESPONDING AUTHOR}

Jonathan P. Winickoff, MD, MPH

Division of General Pediatrics

100 Cambridge St, 15th Floor

Boston, MA 02114

Jwinickoff@mgh.harvard.edu

\begin{abstract}
PURPOSE Conducting studies in national practice-based research networks presents logistic and methodologic challenges. Pediatric Research in Office Settings (PROS) has learned valuable lessons in implementing new strategies and adapting to challenges. We describe practical challenges and results of novel applied strategies in implementing and testing the Clinical Effort Against Secondhand Smoke Exposure (CEASE) intervention as part of a national-level cluster-randomized controlled trial.
\end{abstract}

METHODS In the trial, 20 PROS practices were randomized to either a CEASE intervention arm or a control arm. Parents of children seen in the office who indicated smoking in the past 7 days were asked to complete a postvisit enrollment interview and telephone interviews 3 and 12 months later. Identified challenges included (1) recruiting 20 practices serving a high percentage of parent smokers; (2) screening all parents bringing children for visits and enrolling eligible parents who smoked; and (3) achieving an acceptable 12-month telephone response rate.

RESULTS A total of 47 interested practices completed the Practice Population Survey, of which 20 practices in 16 states completed parent enrollment. Thirty-two research assistants screened 18,607 parents and enrolled 1,980 of them. The initial telephone interview response rate was $56 \%$ at 12 months, with incorrect and disconnected numbers accounting for nearly $60 \%$ of nonresponses. The response rate rose to $67 \%$ after practices supplied 532 new contact numbers and 754 text messages were sent, with 389 parents completing interviews.

CONCLUSION The strategies we used to overcome methodologic barriers in conducting a national intervention trial allowed data collection to be completed in the office setting and increased the telephone interview response rate.

Ann Fam Med 2015;13:475-479. doi: 10.1370/afm.1809.

\section{INTRODUCTION}

$\mathrm{P}$ ediatric Research in Office Settings (PROS), 1 the practice-based research network (PBRN) of the American Academy of Pediatrics, has been conducting studies for 28 years in all 50 states and the Commonwealth of Puerto Rico. Conducting studies in national, as opposed to regional, $\mathrm{PBRNs^{2,3 }}$ presents logistic and methodologic challenges because study procedures need to be followed remotely. In addition, as communication technologies continue to change and health care settings adopt electronic health records, many techniques used traditionally for conducting research across varied primary care settings have become unwieldy and unwelcome. There has been widespread adoption of answering machines, caller ID, and voicemail, and the majority of adults surveyed in 1999 admitted to screening calls. ${ }^{4}$ In addition, mobile telephones are replacing land lines, with $90 \%$ of Americans having a cell phone in $2014^{5}$ and about $41 \%$ of US households having only wireless phones in $2013 .{ }^{6}$ Texting is also replacing conversation and voicemail, ${ }^{7}$ a transition that began a shortly before our study. Adults aged 18 to 44 years used their telephones more for text messages than for calls in $2007 .{ }^{8}$ Among other changes, individuals move frequently, change practices, and change telephone numbers, making 
completion of follow-up calls more difficult..$^{9}$ Finally, with the financial pressures facing primary care, practices are less able to commit their own resources to data collection and other study procedures.

PROS has adapted to these changes to attain practice and participant recruitment, enrollment, and response rate goals. Specifically, novel strategies were needed to address challenges encountered in the PROS Clinical Effort Against Secondhand Smoke Exposure (CEASE) study, a national cluster-randomized controlled trial of parental tobacco cessation assistance delivered in pediatric settings. The aims of the study were to test the effectiveness of the CEASE intervention (1) for increasing clinicians' delivery of evidencebased parental smoking cessation assistance and (2) for altering parental smoking behaviors. ${ }^{10}$

We describe several practical challenges in implementing a cluster-randomized trial on a national level as well as the results of novel applied strategies used in implementing and testing the CEASE intervention. The key challenges encountered were (1) identifying and then recruiting practices with a sufficiently high prevalence of parental smoking ${ }_{i}(2)$ screening all parents in recruited practices and enrolling eligible parents; and (3) achieving an acceptable 12-month telephone response rate. We hope that the strategies described below will assist research networks in conducting outcomes research and practical clinical trials in primary care settings in the future. ${ }^{11}$

\section{METHODS}

The CEASE intervention was developed to assist primary care offices in using evidence-based methods to address parental smoking and to help families become tobacco free. The intervention development process and the specific components have been described. ${ }^{8}$ Practices implemented the CEASE intervention by changing care delivery to address parental smoking (eg, by using quitline referrals, nicotine replacement therapy prescriptions).

For study efficiency, only practices with a sufficiently high prevalence and volume of parent smokers were recruited. Identifying these practices was the first challenge. First, the study team approached PROS practices located in states with a smoking rate of at least $15 \%$ by Centers for Disease Control and Prevention estimates. At this time, 4 practices requiring local institutional review board approval completed the process. Most of the practices were privately owned and therefore covered under the American Academy of Pediatrics' Institutional Review Board. Second, practices that saw at least 50 children per day, of whom 10 had at least 1 parent who smoked, were deemed eligible for randomization. The number of patients and parent smokers seen daily was determined by having practices distribute to all parents of patients seen for 3 consecutive days a Practice Population Survey (PPS), which included a question asking whether the parent had smoked a cigarette (even a puff) in the previous 7 days. The parents placed the completed PPS in a sealed envelope and returned it to the front desk staff. Three days of PPS administration caused a minimal burden on the practices while still giving a rough estimate of the percentage of parent smokers in each practice.

Eligible practices were randomized to either the intervention arm (which implemented the CEASE intervention) or the usual care control arm with delayed intervention. The study team hired research assistants (RAs) within proximity of participating practices to screen all parents exiting the pediatric office after their child's visit and to consent and enroll eligible parents.

Study methods have also been described in detail. ${ }^{10,12-17}$ In brief, all parents of children making any type of visit were approached immediately after the visit by an RA and asked to be interviewed. Those who spoke English, had smoked a cigarette (even a puff) within the past 7 days, were aged 18 years or older, and would have a contact number to be reached over the next year were eligible for enrollment. The interview questions included contact information (e-mail and/ or telephone number), demographics (parent and child age, education, race/ethnicity, insurance status), visit reason, smoking rules in car and/or home, and smoking behaviors. Parents who were eligible and agreed to participate were consented by the RA and asked additional questions from the enrollment survey, which assessed smoking behaviors in more detail, including the parent's smoking level (cigarettes per day) and readiness to quit.

Screening continued until approximately 100 eligible parents were enrolled at each practice. Enrolled parents were contacted again 3 and 12 months after their visit by telephone and received $\$ 10$ and $\$ 25$, respectively, for completing those interviews. Practices received an honorarium of $\$ 100$ for participating.

We calculated frequencies and other descriptive statistics for practice demographics, parent screening and enrollment rates across practices, and methods to increase the response rates.

\section{RESULTS}

\section{Recruiting Eligible Practices}

Budget constraints and the need to maximize efficiency required the study team to use different methods than have been used in previous PROS studies. The need to determine the actual prevalence of parental smokers in practices before expending funding resources to train the practitioners on the study protocol and beginning data 
collection was critical, and the PPS served this purpose. Practices were told before completing the PPS that they would not be eligible to participate if minimum requirements were not met, which occurred for 13 practices.

Of the 477 practices approached, 69 responded yes, 162 responded no, and 246 did not respond. Of the 69 yes practices, 47 completed the PPS. Parents in these 47 practices submitted 7,081 surveys (range $=29$ to 301 per practice) with the response rates ranging from $27 \%$ to $100 \%$ (average $=71 \%$ ).

Reasons for practice noninclusion were not meeting the eligibility criteria, inability to obtain local institutional review board approval, deciding the study would be too difficult to complete in their setting, and staff turnover. Participating practices were from 16 states (Supplemental Appendix, available at http:// www.annfammed.org/content/13/5/475/suppl/DC1); the number of participating practitioners per practice ranged from 3 to 10 . Participating practices did not differ from nonparticipating ones in terms of type (almost 50\% were in pediatric group practices) or setting (about 35\% were located in a suburban setting and $20 \%$ in an urban, not inner city area). Two practices, 1 in each arm, were dropped after enrolling parents for a month because they did not meet predetermined minimum recruitment goals.

\section{Screening and Enrolling Parents}

To obtain accurate assessments of information discussed by the practitioners during the visit, it was necessary to interview parents immediately after their visit and impossible to collect data in advance. Many previous PROS studies had used practice or clinic staff to collect study data, but we determined in a 10 -practice pilot study that the time needed to screen, consent, and enroll parents with this approach exceeded that available to practice staff. The study team therefore had to determine a method that would unburden the practice from recruitment tasks, which resulted in the novel strategy of hiring RAs from a distance who were within proximity of participating practices. Practice staff were required only to indicate to parents when they checked in that they should speak with the RA after the visit. Hiring RAs entailed posting job ads in the local area (eg, on Craigslist, with universities/colleges), reviewing resumes, conducting telephone interviews, processing background checks, creating and routing consultant agreements, and conducting telephone and in-person training. The local RAs were all required to electronically complete the Collaborative Institutional Training Initiative human subjects research training and provide the completion certificate to the study team before conducting interviews. RAs at the first 14 practices were paid $\$ 13$ per hour ${ }_{i}$ however, the amount was decreased to $\$ 11$ per hour for RAs at the remaining practices because of budget constraints. The additional funds needed to pay the RAs ranged from $\$ 2,200$ to $\$ 8,900$ per practice, which was related to number of RAs in the practice and duration of enrollment.

A total of 32 RAs screened 18,607 parents and enrolled 1,980 eligible parents. Screening and enrollment rates varied across practices: the percentage of parents admitting to smoking ranged from $7 \%$ to $42 \%$, the percentage declining screening ranged from $5 \%$ to $40 \%$, and the percentage declining enrollment ranged from $15 \%$ to $58 \%$. The range for the number of parents screened per practice was 307 to 2,173 , while the enrollment period varied from 9 to 106 days. Each of the 20 practices ultimately enrolled approximately 100 parent smokers.

\section{Achieving an Acceptable Response Rate}

Parents were asked to complete telephone interviews 3 and 12 months after their visit. Questions asked on the enrollment survey were also asked during these interviews. As in other PROS studies, 18,19 computer-assisted telephone interviews were completed by the University of South Carolina's Institute for Public Service and Policy Research, which followed standard protocol in regard to number of calls made (minimum of 15) during a specific timeframe (over a 3-week period) and at the selected time of day (participants provided a best time to call).

Unfortunately, presumably because of changes in telephone use, the first response rates for these interviews were far lower than those from previous studies. Early results showed that many calls were consistently not answered or that a telephone answering device was reached. The initial telephone interview response rate was $56 \%$ at 12 months, with incorrect and disconnected numbers accounting for nearly $60 \%$ of nonresponses (Figure 1). We used parental education and child's insurance status as a proxy for parental socioeconomic status and did not find any association between these factors and having incorrect numbers at telephone follow-up time.

On the basis of these results, the study team implemented an array of methods that had not been used in prior PROS studies to increase the response rates. First, practices were asked to obtain updated contact numbers from the charts or by contacting parents, for which they received $\$ 20 /$ hour for up to 40 hours for their time and effort. The University of South Carolina's Institute for Public Service and Policy Research would send a fax to the practice requesting the telephone number for the specific patient, and the practice would fax the form back to the institute. Second, practice staff were enlisted to call nonresponding parents and remind them of the interviews. Last, after 5 unsuc- 
cessful call attempts, up to 3 text messages (before the 4 th, 10th, and 15 th calls) were sent to parents with cell phones to encourage them to call or text back regarding desire to participate. The message read: "At (your child's Dr's office) you signed up for a study. Expect call for last study survey \& complete it for $\$ 25$ or call 800 476-3803." Although the messages were not automated interviewer burden was minimal because the data collection was spread over an extended period.

Practices supplied new numbers (range $=11$ to 43 ; mean $=26.6$; median $=28$ ) or made contact with 532 of 970 unreachable parents (55\%). Regarding texting, 1,274 parents $(71 \%)$ supplied cell phone numbers; of these, $754(59 \%)$ received at least 1 text message. These strategies yielded 389 (40\%) additional interview completions. After the text, only 51 participants (7\%) withdrew; the remainder did not respond to the telephone calls that followed the texts. The final response rate was therefore $67 \%$.

\section{DISCUSSION}

Experience from previously conducted PROS studies as well as various novel methodologic strategies assisted in the successful completion of the CEASE study in 20 PROS practices. Lessons learned from conducting this study will undoubtedly contribute to the success of future studies. When enrolling a specific population, it

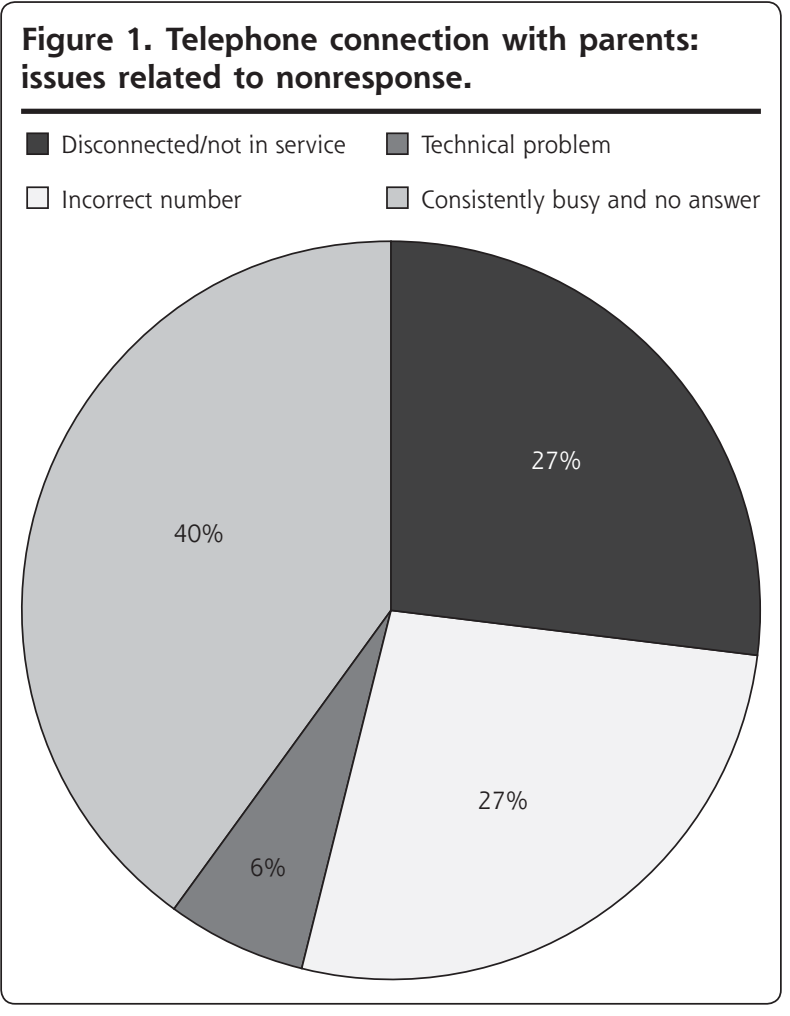

is cost-effective and time efficient to survey potential participating practices beforehand to determine if the sites will provide care to the expected number of participants. The PPS served this purpose for the CEASE study. Unfortunately, solo and small practices were excluded from the study because fewer patients seen per day at a practice resulted in interviews being completed at a slower rate, which cost more money. Also, although using RAs in a practice does not constitute an innovation, the process of hiring and placing RAs in practices from a distance is a novel approach and a challenging solution for any national or large regional PBRN. The strategy of using RAs to complete the screening, consenting, and enrolling of parents allowed the 20 practices to complete the study without practice staff having to worry about enrollment protocols, and allowed practitioners to concentrate on delivering the study intervention and providing care. PROS has determined from surveying network practitioners that the demands of day-today practice leave them with little time to participate in research if the study is complicated and time consuming.

The variation in the RAs regarding parents declining to be screened or enrolled, parents admitting that they smoke, and number of days in the respective enrollment period may have been partially related to distant supervision, but other possible factors were the number of smokers seen at each practice, wait time (eg, parents would not want to extend time at the practice if they had already waited a long time), and seasonality (eg, if the child was sick in winter). Working with the local RAs remotely resulted in more supervision and communication from the study team in the beginning. The RAs typically e-mailed or called study staff only a few times after the initial days, however. Although the strategy of using RAs was successful, it was also costly-an additional lesson learned for planning future work. The financial ramifications are difficult to measure. But we can assume that it was more expensive to hire RAs locally than to have the practice staff complete data collection; however, the study likely would not have been completed if we had relied on practice staff to consent and interview participants. Practices could have been provided a larger honorarium to support existing staff to complete the consenting and interviewing; however, it is difficult to know if doing so would have been successful considering the majority of staff are already employed full time and would therefore have limited available time to perform study tasks. An alternative would be to conduct all parent interviews via telephone, but the results could be potentially low response rates and skewing of information because of participants' recall. By hiring RAs to do the study enrollments, we ensured that practices could focus all of their energy on implementing and sustaining the intervention. 
Although the concurrent implementation of the 3 innovations to increase the 12 -month interview response rate did not allow for comprehensive tracking of the impact of each specific strategy, the combination of innovations yielded a dramatic improvement in response rate in this study. The texting method appears to be feasible in the cell phone era, a potentially cost-saving innovation as well as a way to boost study power. Using alternate communications technology is necessary in this era because, as our data show, many individuals screen out telephone calls and do not listen to voicemails, but they do read and respond to text messages.

In conclusion, the novel strategies we used to overcome methodologic barriers in the conduct of this national PBRN study allowed data collection to be completed in the office setting and increased the telephone interview response rate. All PBRNs will need to continue to adapt to personal lifestyle and practice changes and implement new strategies in order to conduct successful studies going forward.

To read or post commentaries in response to this article, see it online at http://www.annfammed.org/content/13/5/475.

Key words: methodology; secondhand smoke; practice-based research; trial design; follow-up; response rate; pediatrics; primary care; communication

Submitted December 16, 2014; submitted, revised, March 27, 2015; accepted April 28, 2015.

Funding support: This study was supported by the National Institutes of Health $\mathrm{NCl}$ grant R01-CA127127 (to Dr. Winickoff), the National Institute on Drug Abuse, and the Agency for Healthcare Research and Quality. This study was also partially supported by a grant from the Flight Attendant Medical Research Institute to the AAP Julius B. Richmond Center, and the Pediatric Research in Office Settings (PROS) Network receives core funding from the HRSA MCHB (HRSA 5-UA6-10-001) and the AAP.

Acknowledgments: We especially appreciate the efforts of the PROS practices and practitioners. The pediatric practices or individual practitioners who enrolled participants in the larger study are listed here by AAP Chapter: Alaska: Anchorage Pediatric Group, LLC (Anchorage); Connecticut: Hospital of Saint Raphaels (New Haven); Illinois: Community Health Improvement Center (Decatur); Maryland: Cambridge Pediatrics LLC (Waldorf); Massachusetts: Quabbins Pediatrics (Ware), RiverBend Medical Group - Springfield Office (Springfield); Missouri: Priority Care Pediatrics LLC (Kansas City); New Mexico: Las Vegas Clinic for Children and Youth PA (Las Vegas); Ohio: Bryan Medical Group (Bryan), The Cleveland Clinic Wooster (Wooster); Oklahoma: Shawnee Medical Center Clinic (Shawnee); Oregon: Siskiyou Pediatric Clinic LLP (Grants Pass); Pennsylvania: Pennridge Pediatric Associates (Sellersville); South Carolina: Inlet Pediatrics (Murrells Inlet); South Dakota: Avera McGreevy Clinic (Sioux Falls); Tennessee: Raleigh Group PC (Memphis); Virginia: Pediatrics of Kempsville PC (Virginia Beach), Riverside Pediatric Center (Newport News), The Clinic (Richlands); West Virginia: Shenandoah Community Health Center (Martinsburg).

Supplementary materials: Available at http://www.AnnFamMed. org/content/13/5/475/suppl/DC1/.

\section{References}

1. Slora EJ, Wasserman RC. PROS: a research network to enhance practice and improve child health. Pediatr Ann. 2010;39(6):352-361.

2. Agency for Healthcare Research and Quality. Fact Sheet: Primary Care Practice-Based Research Networks. Publication \# 01-P020. http://www.ahrq.gov/research/pbrn/pbrnfact.htm. Last reviewed Dec 2012. Accessed Mar 23, 2015.

3. Slora EJ, Bocian AB, Finch SA, Wasserman RC. Pediatric Research in Office Settings at 25: a quarter century of network research toward the betterment of children's health. Curr Probl Pediatr Adolesc Health Care. 2011;41(10):286-292.

4. Milewski AE. Interruption management and telephone call screening. Int J Hum Comput Interact. 2006;20(1):19-33.

5. Pew Research Center. Mobile Technology Fact Sheet. http://www. pewinternet.org/fact-sheets/mobile-technology-fact-sheet/. Accessed Mar 23, 2015.

6. Pew Research Center. CDC: Two of every five U.S. households have only wireless phones. http://www.pewresearch.org/facttank/2014/07/08/two-of-every-five-u-s-households-have-onlywireless-phones/. Accessed Mar 23, 2015.

7. Pinchot J, Douglas D, Paullet K, Rota D. Talk to text: changing communication patterns. Conference for Information Systems Applied Research; 2011 CONISAR Proceedings. Wilmington, NC: Education Special Interest Group of the AITP; 2011;4(1830):1-9.

8. Nielsen. In U.S., SMS Text Messaging Tops Mobile Phone Calling. http://www.nielsen.com/us/en/insights/news/2008/in-us-textmessaging-tops-mobile-phone-calling.html. Published Sep 22, 2008. Accessed Mar 23, 2015.

9. Kempf AM, Remington PL. New challenges for telephone survey research in the twenty-first century. Annu Rev Public Health. 2007; 28:113-126.

10. Winickoff JP, Nabi-Burza E, Chang Y, et al. Implementation of a parental tobacco control intervention in pediatric practice. Pediatrics. 2013;132(1):109-117.

11. Patient-Centered Outcomes Research Institute. Cooperative Agreement Funding Announcement: Improving Infrastructure for Conducting Patient-Centered Outcomes Research. The National Patient-Centered Clinical Research Network: Clinical Data Research Networks (CDRN)-Phase One. http://www.pcori.org/assets/PCORICDRN-Funding-Announcement-042313.pdf. Released Apr 23, 2013. Accessed Jul 7, 2014.

12. Winickoff JP, Hipple B, Drehmer J, et al. The Clinical Effort Against Secondhand Smoke Exposure (CEASE) intervention: a decade of lessons learned. J Clin Outcomes Manag. 2012;19(9):414-419.

13. Nabi-Burza E, Regan S, Drehmer J, et al. Parents smoking in their cars with children present. Pediatrics. 2012;130(6):e1471-e1478.

14. Drehmer JE, Ossip DJ, Rigotti NA, et al. Pediatrician interventions and thirdhand smoke beliefs of parents. Am J Prev Med. 2012;43 (5):533-536.

15. Ossip DJ, Chang Y, Nabi-Burza E, et al. Strict smoke-free home policies among smoking parents in pediatric settings. Acad Pediatr. 2013;13(6):517-523.

16. Nabi-Burza E, Winickoff JP, Finch S, Regan S. Triple tobacco screen: opportunity to help families become smokefree. Am J Prev Med. 2013;45(6):728-731.

17. Friebely J, Rigotti NA, Chang Y, et al. Parent smoker role conflict and planning to quit smoking: a cross-sectional study. BMC Public Health. 2013;13:164.

18. Barkin SL, Finch SA, Ip EH, et al. Is office-based counseling about media use, timeouts, and firearm storage effective? Results from a cluster-randomized, controlled trial. Pediatrics. 2008;122(1):e15-e25.

19. Resnicow K, McMaster F, Woolford S, et al. Study design and baseline description of the BMI2 trial: reducing paediatric obesity in primary care practices. Pediatr Obes. 2012;7(1):3-15. 